\title{
Diferencias asociadas al sexo en las puntuaciones total y factoriales del Purpose- In-Life Test en universitarios españoles*
}

\author{
Sex differences on Purpose-In-Life Test total and \\ factorial scores among spanish undergraduates
}

Recibido: enero 12 de 2010

Revisado: agosto 14 de 2010

Aceptado: noviembre 23 de 2010

\author{
JOAQUÍN GARCÍA-ALANDETE ** \\ Eva Rosa MARTÍNEZ *** \\ BEATRIZ SOUCASE LOZANO ***** \\ José Francisco GALLEGO-PÉREZ ${ }^{* * * * * *}$ \\ Universidad Católica de Valencia, España
}

Para citar este artículo. García-Alandete, J., Martínez, E. R., Soucase Lozano, B. \& Gallego-Pérez, J. F. (2011). Diferencias asociadas al sexo en las puntuaciones total y factoriales del Purpose-In-Life Test en universitarios españoles. Universitas Psychologica, 10 (3), 681-692.

* Artículo de investigación original en Psicología Diferencial.

** Departamento de Metodología, Psicología Básica y Psicología Social. Facultad de Psicología y Ciencias de la Salud.E-mail: ximo.garcia@ucv.es

**** Departamento de Metodología, Psicología Básica y Psicología Social. Facultad de Psicología y Ciencias de la Salud. E-mail: eva.rosa@ucv.es

***** Departamento de Ciencias de la Ocupación, Psicología Evolutiva y de la Educación. Facultad de Psicología y Ciencias de la Salud. E-mail: beatriz. soucase@ucv.es

******* Departamento de Personalidad, Evaluación y Tratamientos. Facultad de Psicología y Ciencias de la Salud.E-mail: francisco.gallego@ucv.es

\section{RES UMEN}

Se analizan las diferencias en las puntuaciones total y factoriales de la versión española del Purpose-In-Life Test [PIL] (Crumbaugh \& Maholic, 1969; Noblejas de la Flor, 1994) asociadas al sexo, en un grupo de 309 estudiantes universitarios (207 mujeres y 102 hombres) de edades comprendidas entre 18 y 45 años. El PIL evalúa logro de sentido de la vida vs. vacío existencial. Las mujeres obtienen puntuaciones medias superiores a los hombres, tanto en la puntuación total del PIL como en sus diferentes factores. El análisis estadístico muestra que el sexo da lugar a diferencias estadísticamente significativas en la puntuación total y en los factores Percepción de sentido y Metas y tareas del PIL.

Palabras clave autores

Sexo, sentido de la vida, Purpose-In-Life Test, PIL.

Palabras clave descriptores

Pruebas psicológicas, validación, investigación cuantitativa.

\section{A B S T R A C T}

The aim of this paper is to analyze the differences on Purpose-In-Life Test [PIL] (Crumbaugh \& Maholic, 1969) total and factorial scores associated to sex, among 309 spanish undergratudates (207 women, 102 men), range 18 to 45 years. PIL Spanish version is used (Noblejas de la Flor, 1994). PIL evalues life meaning achievement vs. existential vacuum. Women achieve higher means on PIL total and factorial scores, and estatistical analysis show that sex is significantly associated to total PIL score and on Purpose perception and Goals and tasks factors scores.

Key words authors

Sex, meaning in life, Purpose-In-Life Test, PIL.

Key words plus

Psychologycal test, validation, quantitative research. 
En las últimas décadas, un cuerpo creciente de investigación ha dedicado su atención al constructo sentido de la vida (meaning in life), uno de los supuestos fundamentales de la logoterapia de Frankl (1994; Lukas y Hirsch, 2002). La voluntad de sentido (will to meaning) sería para estos autores la principal motivación del ser humano, motivación de orden existencial, universal y no dependiente de variables demográficas, tales como la edad, el sexo, el estatus socioeconómico, el nivel de formación académica y otras. Se trata de una necesidad existencialmente prioritaria a cualquier otra, sea biológica (salud, fortaleza, sexo), psicológica (bienestar subjetivo) o social (prestigio, éxito) consistente en encontrar un motivo de orden existencial por el cual, pese incluso a toda circunstancia adversa, la vida es percibida y experimentada como valiosa (logro de sentido). Este constructo ha obtenido una clara fundamentación empírica a través de instrumentos de evaluación que cumplen los debidos requisitos psicométricos (c.f. Chamberlain \& Zika, 1988; Lukas, 1986; Schulenberg, 2003).

Como se ha señalado, según Frankl (1994), la posibilidad de logro de sentido de la vida es universal e independiente de las características sociodemográficas de los individuos. Pero esto es distinto a que tales diferencias puedan suponer, a su vez, diferencias en el nivel de logro de sentido de la vida. En relación con eso, se han llevado a cabo estudios que intentan comprobar si existen diferencias significativas interindividuales en las puntuaciones de los instrumentos que evalúan el logro de sentido de la vida, principalmente el Purpose-In-Life Test [PIL] (Crumbaugh \& Maholick, 1969), que es el utilizado en la presente investigación, en su versión castellana (Noblejas de la Flor, 1994).

Por otra parte, como es sabido, el estudio de las diferencias asociadas al sexo, como variable sujeto o independiente, es recurrente en los estudios psicológicos desde hace décadas, sobre todo en el área de la Psicología Diferencial y en diversos ámbitos específicos de investigación, tales como el razonamiento lógicomatemático, los estilos cognitivos, la inteligencia general, la competencia visoespacial, la personalidad, el razonamiento moral, la empatía y la prosocialidad, entre otros (cfr. Barberá, 1998; Fernández, 1988, 1996; Jayme \& Sau, 1996; Maccoby \& Jacklin, 1974; Martínez \& Bonilla, 2000; Unger, 1979), siendo un área de difícil y polémica investigación, dada la intervención de importantes factores de naturaleza sociocultural, tales como normas y roles sociales (García-Vega, Fernández \& Rico, 2005). Algunos de estos trabajos, de manera directa o indirecta según sus objetivos específicos, analizan la posible influencia del sexo sobre la puntuación total del PIL, siendo los resultados dispares $y$, en consecuencia, poco concluyentes.

Así, Crumbaugh y Maholick (1964), con una muestra compuesta por 225 sujetos distribuidos en 5 grupos, tres de ellos clínicos y dos normales (30 sujetos con alto sentido de la vida, 75 estudiantes universitarios, 49 pacientes externos de consultas privadas de Psiquiatría, 50 pacientes externos de un centro psiquiátrico, 21 alcohólicos hospitalizados; todos los grupos incluían hombres y mujeres), hallaron que, si bien en todos los grupos las mujeres obtenían puntuaciones más altas en la puntuación total del PIL y en la mayor parte de ítems que los hombres, las diferencias no eran estadísticamente significativas en ninguno de ellos.

En un estudio de Meier y Edwards (1974) con un grupo de 200 sujetos (entre 13 y más de 65 años), pertenecientes a distintas comunidades religiosas (anglicana, baptista, luterana, católica y de la Iglesia Unida), los hombres alcanzaron una media $(M=109 ; D T=15)$ superior a la de las mujeres $(M=107.8 ; D T=14)$ en la puntuación total del PIL, si bien esta diferencia no resultó estadísticamente significativa.

Sallee y Casciani (1976), con un grupo de 48 universitarios (18 hombres, 30 mujeres; edad media 23.9 años), hallaron que, si bien las mujeres obtenían una media superior que los hombres en la puntuación total del PIL (mujeres: $M=107.43$; $D T=14.27$; hombres: $M=102.22 ; D T=16.59$ ), las diferencias no resultaron estadísticamente significativas. Tampoco Coffield y Buckalew (1986) hallaron diferencias estadísticamente significativas asociadas al sexo en la puntuación del PIL, en un grupo de 230 estudiantes universitarios, ni Jackson y Coursey (1988), con una muestra compuesta por 
98 miembros de una comunidad baptista negra de Washington (70 mujeres y 30 hombres, entre 1868 años y 38.5 años de edad media).

Noblejas de la Flor (1994), en una investigación con 841 sujetos (344 hombres, 492 mujeres, 5 sin datos sobre su sexo; rango de edades desde menores de 20 años a mayores de 60), encontró que la puntuación total media en el PIL era independiente del sexo. Sivberg (2002) tampoco encontró diferencias en la puntuación del PIL asociadas al sexo, utilizando como estadístico la prueba $t$ de Student para muestras independientes, en un grupo de 66 padres de niños con trastornos del espectro autista y en un grupo de control formado por 66 padres de niños sin este tipo de trastornos.

Gallego-Pérez y García-Alandete (2004), con una muestra de 99 universitarios (31 hombres, 68 mujeres; edad media $=20.96$ años) no hallaron diferencias significativas en la puntuación total del PIL, como tampoco García-Alandete, GallegoPérez y Pérez-Delgado (2009) con una muestra de 302 universitarios (99 hombres y 203 mujeres; edad media $=21.58$ años) en la misma puntuación. El mismo resultado de no significatividad de las diferencias por sexo hallaron Flood y Boyd (2008) en un grupo de 57 personas mayores (entre $57 \mathrm{y}$ 94 años).

Por el contrario, en un estudio, ya clásico, cuyo objetivo consistía en establecer la validez de constructo y concurrente del PIL, Crumbaugh (1968) halló, en un grupo 602 hombres y 543 mujeres (de una muestra total de 1151 sujetos), diferencias significativas asociadas al sexo $(t=4.08 ; p<$ 0.001 ), obteniendo los hombres una puntuación media más alta que las mujeres (hombres: $\mathrm{M}=$ 108.68; $D T=18.10$; mujeres: $M=104.10 ; D T=$ 19.66). El investigador señalaba que este resultado era contrario a investigaciones precedentes (en las que, si bien las diferencias no eran estadísticamente significativas, sí eran consistentes), pudiéndose deber a una representación sensiblemente mayor de hombres que de mujeres en uno de los grupos (214 hombres, 16 mujeres), con una ratio muy superior a la del resto de grupos.

También Doerries (1970) con una muestra de 184 estudiantes de Psicología, halló una diferencia estadísticamente significativa $(p<0.01)$, alcanzando las mujeres una puntuación media más alta que los hombres. Por su parte, Mak y Shek (1990), con un grupo de 1.000 sujetos (500 hombres y 500 mujeres, entre 20 y 40 años), hallaron que el sexo se asociaba a diferencias estadísticamente significativas en las puntuaciones medias del PIL $\left(F_{(1,792)}=\right.$ 27.32; $p<0.001)$, siendo la de los hombres $(M=$ 99.28) más alta que la de las mujeres $(M=91.82)$.

En otro estudio, Nygren et al. (2005) con un grupo de 125 personas mayores de 85 años ( $86 \mathrm{mu}$ jeres, 39 hombres) en el que los hombres alcanzaron una puntuación media del PIL media más alta (M $=110 ; \mathrm{DT}=13.3)$ que las mujeres $(\mathrm{M}=103$; $D T=16.3)$, esta diferencia fue estadísticamente significativa $(p=0.017)$. Molasso (2006) con 354 estudiantes de secundaria (234 mujeres, 120 hombres; edad media) y utilizando ANOVA como prueba estadística, también halló diferencias significativas en la puntuación total del PIL asociadas al sexo $\left(F_{(1,357)}=3.99 ; p<0.05\right)$, siendo las mujeres quienes alcanzaron puntuaciones superiores.

Considerando lo expuesto, una cuestión relevante es la divergencia en los resultados de las investigaciones, estando lejos de ser claras las relaciones entre sexo y logro de sentido de la vida, tal como es medido a través del PIL, y sugiriendo la pertinencia de seguir investigando. Con todo, debe tenerse en cuenta la heterogeneidad de las muestras utilizadas en los estudios reseñados. A esto debe añadirse, como aspecto a subrayar del presente trabajo, que las investigaciones precedentes se han centrado en la puntuación total del PIL, dejando fuera de análisis los distintos factores de este instrumento (Noblejas de la Flor, 1994; Reker, 2000; Reker \& Chamberlain, 2000), a excepción del trabajo de Gallego-Pérez, García-Alandete y Pérez-Delgado (2007) sobre las relaciones entre religiosidad y sentido de la vida.

Con el fin de aportar datos que puedan resultar de interés y sumar evidencias empíricas, así como estimular futuras investigaciones, el presente trabajo tiene como objetivo comprobar si se asocian diferencias al sexo, en qué sentido se dan y si resultan estadísticamente significativas, tanto en la puntuación total como en las puntuaciones facto- 
riales del PIL, como medidas del logro de sentido de la vida y de aspectos específicos del mismo, respectivamente.

\section{Método}

\section{Participantes y procedimiento}

La muestra está compuesta por 309 universitarios, 207 mujeres (66.99\%) y 102 hombres $(33.01 \%)$, con edades entre 18 y 45 años $(M=21.4 ; D T$ $=3.254$ ), de la ciudad de Valencia (España), que aceptaron participar voluntariamente en la presente investigación. Los participantes debían cumplimentar un protocolo que solicitaba datos sociodemográficos e incluía la versión castellana del PIL. Los protocolos incorrecta o incompletamente cumplimentados se desecharon, introduciéndose los datos de los válidos en un archivo Excel, siendo posteriormente exportados a una hoja de cálculo SPSS 17.0 para Windows, programa con el que se han realizado los análisis estadísticos, concretamente, la prueba $t$ de Student para muestras independientes.

\section{Instrumento de evaluación}

Como instrumento de evaluación se utilizó la parte A del Purpose-in-Life Test [PIL] (Crumbaugh \& Maholick, 1969), en su versión castellana (Noblejas de la Flor, 1994). Se trata de una escala tipo Likert (1 a 7) de 20 items, cuya puntuación se obtiene sumando los valores de las opciones de respuesta que el sujeto selecciona, permitiendo obtener una medida del logro de sentido de la vida desde planteamientos logoterapéuticos (Crumbaugh, 1968; Crumbaugh \& Maholick, 1964). Alcanza un coeficiente de fiabilidad de 0.84 (Guttmann, 1996), razonables validez de constructo y concurrente (Crumbaugh \& Henrion, 1988) y ha sido sometido a estudios de validación cruzada (Crandall \& Rasmussen, 1975; Crumbaugh, Raphael \& Shrader, 1970). La versión castellana (véase Apéndice) ha mostrado tener una alta consistencia interna (alfa de Cronbach $=0.88)$. La interpretación de la Parte A del PIL, según los datos de la baremación de Noblejas de la Flor (1994), es la siguiente: puntuación < 90: vacío existencial; puntuación entre 90 y 105: indefinición con respecto al sentido de la vida; puntuación $>105$ : presencia de metas y sentido de la vida. Por otro lado, el análisis factorial de la versión castellana de esta parte del PIL distingue cuatro factores, que explican el $54 \%$ de la varianza en el espacio de los valores y el $100 \%$ en el factorial (Noblejas de la Flor, 1994).

Percepción de Sentido: captación de motivos y razones para vivir la vida y la valoración que esta, en general, merece, explica el $35.9 \%$ de la varianza y está compuesto por los items 4, 6, 9, 10, 11, 12, 16,17 y 20 del PIL, pudiendo oscilar la puntuación entre 9 y 56.

Experiencia de Sentido: percepción de la vida propia y de la vida cotidiana como plenas de cosas buenas, explica el $6.8 \%$ de la varianza e incluye los ítems 1, 2, 5, 9, 17, 19 y 20 del PIL, pudiendo oscilar la puntuación entre 7 y 49 .

Metas y Tareas: objetivos ligados a acciones concretas en la vida y a la responsabilidad personal percibida hacia los mismos, explica el $5.8 \%$ de la varianza y está integrado por los items 3, 7, 8, 13, 17,19 y 20 del PIL, pudiendo oscilar la puntuación entre 7 y 49.

Dialéctica Destino/Libertad: tensión destino vs. libertad y afrontamiento de la muerte como acontecimiento incontrolable, impredecible e inevitable, explica el $5.5 \%$ de la varianza e incluye los items 14, 15 y 18 del PIL, pudiendo oscilar la puntuación entre 3 y 21.

\section{Resultados}

Previamente al análisis de la proporción de varianza que el sexo explica de las puntuaciones total y factoriales del PIL, mediante la prueba de comparación de medias para muestras independientes, se calculan las medias y desviaciones típicas de mujeres y hombres. Como se observa en la Tabla 1 , las mujeres alcanzan medias superiores a las de los hombres en todas las puntuaciones.

La probabilidad asociada al estadístico de Levene, mayor que 0.05 para todas las puntuaciones del PIL, permite suponer la homogeneidad de 
TABLA 1

Estadísticos de grupo

\begin{tabular}{lccccc}
\hline & Sexo & $N$ & Media & Desviación típica & $\begin{array}{c}\text { Error típico de la } \\
\text { media }\end{array}$ \\
\hline PIL Total & Mujeres & 207 & 110.4928 & 14.44772 & 1.00419 \\
& Hombres & 102 & 106.5882 & 13.69639 & 1.35614 \\
\hline Percepción de sentido & Mujeres & 207 & 51.4251 & 7.88282 & 0.54789 \\
& Hombres & 102 & 49.5000 & 7.33600 & 0.72637 \\
\hline Experiencia de sentido & Mujeres & 207 & 38.3527 & 5.96318 & 0.41447 \\
& Hombres & 102 & 37.2941 & 5.42380 & 0.53704 \\
\hline Metas y tareas & Mujeres & 207 & 40.8599 & 4.47481 & 0.31102 \\
& Hombres & 102 & 38.9510 & 5.02149 & 0.49720 \\
\hline Dialéctica destino/libertad & Mujeres & 207 & 14.6184 & 3.05678 & 0.21246 \\
& Hombres & 102 & 14.0980 & 2.96685 & 0.29376 \\
\hline
\end{tabular}

Fuente: elaboración propia.

varianzas poblacionales (Tabla 2). Por su parte, la prueba $t$ de Student muestra que las diferencias entre hombres y mujeres alcanzan la significatividad estadística en la puntuación total $(t=2.272 ; p=$ 0.024) y en los factores Percepción de Sentido ( $t$ $=2.065 ; p=0.040)$ y Metas y Tareas $(t=3.385$; $p=0.001)$. Los límites del intervalo de confianza (a1 $95 \%$ ) permiten estimar que la diferencia media entre hombres y mujeres es mayor en la puntuación total del PIL, seguida de la puntuación del factor Percepción de Sentido y, en tercer lugar, del factor Metas y Tareas.

\section{Discusión}

El objetivo de este trabajo era comprobar si el sexo se asocia a diferencias significativas en las puntuaciones del PIL, estando esto motivado por la disparidad de resultados al respecto en investigaciones previas (en relación con la puntuación total) y ausencia de investigaciones (en el caso de las puntuaciones factoriales). Para ello se lleva a cabo una prueba de contraste de medias, concretamente una prueba $t$ de Student para muestras independientes, cuyos resultados muestran que el sexo explica de manera estadísticamente significativa la diferencia de medias entre hombres y mujeres en la puntuación total del PIL $(t=2.272 ; p=0.024)$ y en los factores Percepción de Sentido $(t=2.065 ; p$ $=0.040)$ y Metas y Tareas $(t=3.385 ; p=0.001)$.

En el grupo estudiado y de manera estadísticamente significativa, las mujeres se caracterizan por un nivel general de sentido de la vida superior al de los hombres, por mayor percepción de sentido y valoración de la vida como llena de cosas buenas, emocionantes y razones para ser vivida, por una percepción más positiva del mundo en relación con la propia vida, por tener objetivos y metas ligados a las acciones concretas cotidianas, por un mayor sentido de responsabilidad hacia los objetivos existenciales y por dedicar menos pensamientos al suicidio. Asimismo, son más sensibles a las cosas de la vida cotidiana y les atribuyen más importancia, muestran un mayor sentido de responsabilidad con respecto al día a día y con respecto a las metas y tareas más inmediatas, concretas y específicas.

En cuanto a la significatividad estadística de las diferencias en la puntuación total del PIL asociadas al sexo, los resultados son coincidentes con Crumbaugh (1968), Doerries (1970), Molasso (2006), Mak y Shek (1990) y Nygren et al. (2005), si bien en el primero y en los dos últimos trabajos las pun- 
TABLA 2

Prueba de muestras independientes

\begin{tabular}{|c|c|c|c|c|c|c|c|c|c|c|}
\hline & & \multirow{2}{*}{\multicolumn{2}{|c|}{$\begin{array}{c}\text { Prueba de } \\
\text { Levene para } \\
\text { la igualdad de } \\
\text { varianzas }\end{array}$}} & \multicolumn{7}{|c|}{ Prueba $t$ para la igualdad de medias } \\
\hline & & & & \multirow[b]{2}{*}{$t$} & \multirow[b]{2}{*}{$g l$} & \multirow[b]{2}{*}{$\begin{array}{c}\text { Sig. } \\
\text { (bilateral) }\end{array}$} & \multirow[b]{2}{*}{$\begin{array}{l}\text { Diferencia } \\
\text { de medias }\end{array}$} & \multirow[b]{2}{*}{$\begin{array}{l}\text { Error típico de } \\
\text { la diferencia }\end{array}$} & \multicolumn{2}{|c|}{$\begin{array}{c}95 \% \text { Intervalo de } \\
\text { confianza para la } \\
\text { diferencia }\end{array}$} \\
\hline & & F & Sig. & & & & & & Inferior & Superior \\
\hline \multirow[t]{2}{*}{$\begin{array}{l}\text { PIL } \\
\text { Total }\end{array}$} & $\begin{array}{l}\text { Se han asumi- } \\
\text { do varianzas } \\
\text { iguales }\end{array}$ & 0.001 & 0.979 & 2.272 & 307 & 0.024 & 3.90452 & 1.71843 & 0.52312 & 7.28592 \\
\hline & $\begin{array}{l}\text { No se han asu- } \\
\text { mido varian- } \\
\text { zas iguales }\end{array}$ & & & 2.314 & 211.017 & 0.022 & 3.90452 & 1.68746 & 0.57808 & 7.23096 \\
\hline \multirow[t]{2}{*}{$\begin{array}{l}\text { Percep- } \\
\text { ción de } \\
\text { Sentido }\end{array}$} & $\begin{array}{l}\text { Se han asumi- } \\
\text { do varianzas } \\
\text { iguales }\end{array}$ & 0.423 & 0.516 & 2.065 & 307 & 0.040 & 1.92512 & 0.93238 & 0.09047 & 3.75978 \\
\hline & $\begin{array}{l}\text { No se han asu- } \\
\text { mido varian- } \\
\text { zas iguales }\end{array}$ & & & 2.116 & 214.568 & 0.036 & 1.92512 & 0.90984 & 0.13176 & 3.71848 \\
\hline \multirow[t]{2}{*}{$\begin{array}{l}\text { Expe- } \\
\text { riencia } \\
\text { de Sen- } \\
\text { tido }\end{array}$} & $\begin{array}{l}\text { Se han asumi- } \\
\text { do varianzas } \\
\text { iguales }\end{array}$ & 0.366 & 0.546 & 1.511 & 307 & 0.132 & 1.05854 & 0.70060 & -0.32004 & 2.43712 \\
\hline & $\begin{array}{l}\text { No se han asu- } \\
\text { mido varian- } \\
\text { zas iguales }\end{array}$ & & & 1.560 & 219.048 & 0.120 & 1.05854 & 0.67838 & -0.27844 & 2.39552 \\
\hline \multirow[t]{2}{*}{$\begin{array}{l}\text { Metas y } \\
\text { Tareas }\end{array}$} & $\begin{array}{l}\text { Se han asumi- } \\
\text { do varianzas } \\
\text { iguales }\end{array}$ & 3.144 & 0.077 & 3.385 & 307 & 0.001 & 1.90892 & 0.56395 & 0.79922 & 3.01863 \\
\hline & $\begin{array}{l}\text { No se han asu- } \\
\text { mido varian- } \\
\text { zas iguales }\end{array}$ & & & 3.255 & 181.856 & 0.001 & 1.90892 & 0.58647 & 0.75177 & 3.06608 \\
\hline \multirow[t]{2}{*}{$\begin{array}{l}\text { Dialécti- } \\
\text { ca desti- } \\
\text { no/ } \\
\text { Libertad }\end{array}$} & $\begin{array}{l}\text { Se han asumi- } \\
\text { do varianzas } \\
\text { iguales }\end{array}$ & 0.255 & 0.614 & 1.421 & 307 & 0.156 & 0.52032 & 0.36625 & -0.20036 & 1.24099 \\
\hline & $\begin{array}{l}\text { No se han asu- } \\
\text { mido varian- } \\
\text { zas iguales }\end{array}$ & & & 1.435 & 206.584 & 0.153 & 0.52032 & 0.36254 & -0.19444 & 1.23507 \\
\hline
\end{tabular}

Fuente: elaboración propia.

tuaciones medias más altas fueron alcanzadas por los hombres y no por las mujeres, contrariamente a lo obtenido en el presente estudio. Por otra parte, como se señaló más arriba, hay que tener en cuenta la heterogeneidad en la composición de las muestras entre los estudios revisados, y entre estos y la del presente, lo cual exige cautela en los contrastes de resultados. 
Debe subrayarse que, en relación con las puntuaciones factoriales, se carece de precedentes con los que poder contrastar los resultados obtenidos, por lo que el estudio presente supone una aportación original en la investigación con el PIL, en particular, y en la investigación sobre el logro de sentido de la vida, en general. Solo en uno de los trabajos revisados se tuvieron en cuenta los factores del PIL, en relación con la religiosidad (GallegoPérez, García-Alandete \& Pérez-Delgado, 2007), pero no hay antecedentes en los que se analice la relación entre el sexo y tales factores. $\mathrm{Al}$ respecto, los resultados de este estudio sugieren el interés de considerar no solo las puntuaciones totales de los instrumentos de medida, sino también, si es el caso, las puntuaciones factoriales, cuando se mide el posible influjo de variables clásicamente relevantes en los estudios psicológicos, ya que pueden existir diferencias significativas para las que las puntuaciones totales no son suficientemente sensibles, haciéndose necesario afinar los análisis considerando aquéllas. La consideración de las puntuaciones factoriales del PIL en relación con el sexo es, pues, un aspecto relevante de este trabajo, por su originalidad y porque ha posibilitado la obtención de datos que, de otro modo, pasarían inadvertidos y que resultan de interés por sí mismos, toda vez que pueden estimular futuras investigaciones, relativas a, por ejemplo, si el constructo sentido de la vida tiene en su base procesos psicológicos, estilos cognitivo-emocionales y/o características de personalidad que permitan explicar las diferencias entre hombres y mujeres en percepción y logro de sentido, valoración de la vida y establecimiento y responsabilidad hacia metas y tareas cotidianas.

En relación con esto último, sugerimos que tales diferencias son, fundamentalmente, de orden cognitivo-perceptual, considerando que, desde la misma logoterapia se ha sostenido que la voluntad de sentido puede entenderse en términos de las leyes organizativas perceptuales formuladas por la Gestalt (Crumbaugh, 1973). La voluntad de sentido sería, primariamente, un fenómeno perceptual, consistiendo concretamente en la capacidad de percibir y atribuir sentido al ambiente, de interpretar y organizar los estímulos con el fin de construir totalidades llenas de sentido. La percepción de sentido sería un tipo específico de categorización e interpretación perceptual innata del ser humano, con valor adaptativo. Esta percepción de sentido sería relativa a una justificación para la propia existencia, al logro de sentido de la vida, pudiéndose hablar entonces de percepción del sentido de la vida ("iQué sentido tiene esto que me ha sucedido?”, “¿Qué sentido encierra esta situación, este problema, este suceso, esta circunstancia que acontece en mi vida?").

Siendo una categoría fundamentalmente perceptual, la voluntad de sentido podría estar estrechamente relacionada con el estilo cognitivo Dependencia/Independencia de Campo [DIC] (Witkin \& Goodenough, 1985). Las diferencias entre hombres y mujeres en percepción de sentido se deberían a diferencias en el estilo DIC, por otra parte ya constatadas en otras investigaciones (Witkin \& Goodenough, 1985). Más concretamente, las mujeres se caracterizan por un modo perceptivo DC, mientras que los hombres por un modo perceptivo IC. Y si la DC se asocia a la influencia que experimenta la percepción del sujeto por la organización del campo que rodea un elemento (algún acontecimiento o circunstancia cotidiana, por ejemplo), a "una tendencia a agrupar los objetos en función de una determinada característica, porque el fondo ejerce un gran influencia perceptiva capaz de articular los elementos que contiene" (Jayme \& Sau, 1996, p. 202), a una percepción holística, esto podría estar asociado a una capacidad perceptual gestáltica, a una sensibilidad específica para la percepción del sentido de la vida, característica de las mujeres en mayor medida que de los hombres. Las mujeres, al caracterizarse cognitivamente por la DC, tendrían una mayor capacidad para percibir el potencial sentido de las situaciones cotidianas, al percibirlas enmarcadas en un todo existencial significativo. Los hombres, por el contrario, se caracterizarían por la IC, siendo más analíticos y con menor capacidad para percibir los elementos contenidos en un contexto, situación o circunstancia en un conjunto de sentido global y unitario. Si esta hipótesis fuera correcta (la percepción de sentido de la vida se asocia positiva y significativamente a 
la DC y, a su vez, esto explica las diferencias entre hombres y mujeres en aquélla), debería esperarse, en los estudios que a tal efecto se llevaran a cabo, una significativa asociación positiva entre la $\mathrm{DC}$ y puntuaciones más altas en el PIL, tanto total como en el factor Percepción de Sentido, en particular, sin que ello no significase que se dieran diferencias en el resto de factores.

Conscientes de lo arriesgada que es la hipótesis expuesta (no explica, por ejemplo, los resultados de los trabajos en los que han sido los hombres quienes obtuvieron puntuaciones más altas en el PIL), consideramos que es razonable, toda vez que perfectamente contrastable, planteando una interesante línea de investigación, al día de hoy por desarrollar, y que esperamos haber contribuido a estimular, sobre las relaciones entre la voluntad de sentido y otras variables, tales como los estilos cognitivos y, en particular, el estilo DIC.

\section{Referencias}

Barberá, E. (1998). Psicología del género. Barcelona: Ariel.

Chamberlain, K. \& Zika, S. (1988). Measuring meaning in life: An examination of three scales. Personality and Individual Differences, 9, 589-596.

Coffield, K. E. \& Buckalew, L. W. (1986). Student apathy: An analysis of relevant variables. College Student Journal, 20, 211-214.

Crandall, J. E. \& Rasmussen, R. D. (1975). Purpouse In Life as related to specific values. Journal of Clinical Psychology, 31, 483-485.

Crumbaugh, J. C. (1968). Cross-validation of Purpose in Life Test based on Frankl's concepts. Journal of Individual Psychology, 24, 74-81.

Crumbaugh, J. C. (1973). The validation of logotherapy. En R. M. Jurjevich (Dir.), Direct Psychotherapy (pp. 34-76). University of Miami, FL: Coral Glabes.

Crumbaugh, J. C. \& Henrion, R. (1988). The PIL Test: Administration, interpretation, uses theory and critique. International Forum for Logotherapy, 11 (2), 76-88.

Crumbaugh, J. C. \& Maholick, L. T. (1964). An experimental study in existentialism: The psychometric approach to Frankl's concept of noogenic neurosis. Journal of Clinical Psychology, 20, 589-596.

Crumbaugh, J. C. \& Maholick, L. T. (1969). Manual of instructions for the Purpose in Life Test. Saratoga: Viktor Frankl Institute of Logotherapy.

Crumbaugh, J. C., Raphael, S. M. \& Shrader, R. R. (1970). Frankl's view to meaning in a religious order. Journal of Clinical Psychology, 26, 206-207.

Doerries, L. E. (1970). Purpose in life and social participation. Journal of Individual Psychology, 26, 50-53.

Fernández, J. (1996). Varones y mujeres. Desarrollo de la doble realidad del sexo y del género. Madrid: Pirámide.

Fernández, J. (Coord.). (1988). Nuevas perspectivas en el desarrollo del sexo y el género. Madrid: Pirámide.

Flood, M. \& Boyd, M. (2008). Successful aging in a southern older adult sample. Southern Online Journal of Nursing Research, 8 (3). Consultado el 25 de marzo, 2009, en http://snrs.org/publications/ SOJNR_articles2/Vol08Num03Art06.html

Frankl, V. E. (1994). Logoterapia y análisis existencial. Barcelona: Herder.

Gallego-Pérez, J. F. \& García-Alandete, J. (2004). Sentido en la vida y desesperanza en un grupo de estudiantes universitarios. NOUS. Boletín de Logoterapia y Análisis Existencial, 8, 49-64.

Gallego-Pérez, J. F., García-Alandete, J. \& Pérez-Delgado, E. (2007). Factores del Purpose In Life y religiosidad. Universitas Psychologica, 6 (2), 213-229.

García-Alandete, J., Gallego-Pérez, J. F. \& Pérez-Delgado, E. (2009). Sentido de la vida y desesperanza: un estudio empírico. Universitas Psychologica, 8 (2), 447-454.

García-Vega, E., Fernández, P. \& Rico, A. (2005). Género y sexo como variables moduladoras del comportamiento sexual en jóvenes universitarios. Psicothema, 17 (1), 49-56.

Guttmann, D. (1996). Logotherapy for the helping profesional: Meaninful social work. New York: Springer Publishing.

Jackson, L. E. \& Coursey, R. D. (1988). The relationship of God control and internal locus of control to intrinsic religious motivation, coping and purpose in life. Journal for the Scientific Study of Religion, 27 (3), 399-410. 
Jayme, M. \& Sau, V. (1996). Psicología diferencial del sexo y el género. Fundamentos. Barcelona: Icaria.

Lukas, E. \& Hirsch, B. Z. (2002). Logotherapy. En R. F. Massey \& S. D. Massey (Eds.), Comprehensive handbook of psychotherapy: Interpersonal/Humanistic/Existential (Vol. 3, pp. 333-356). New York: John Wiley \& Sons.

Lukas, E. (1986). LOGO-test. Test zur Messung von 'innerer Sinnerfüllung' und 'existentieller Frustration'. Wien: Deuticke. [LOGO-TEST. Test para la medición de la 'realización interior de sentido' y de la 'frustración existencial' (C. Piechocki, Trad.). Buenos Aires: Almagesto, 1996.]

Maccoby, E. E. \& Jacklin, C. N. (1974). The Psychology of sex differences. Stanford, CA: Stanford University Press.

Mak, W. -K. \& Shek, D. T. L. (1990). Sex differences in the psychological well-being of Chinese dualemployed parents in Hong Kong. CUHKEducation Journal, 18 (2), 115-120.

Martínez, I. \& Bonilla, A. (2000). Sistema sexo/género, identidades y construcción de la subjetividad. Valencia: Publicacions de la Universitat de València.

Meier, A. \& Edwards, H. (1974). Purpose-In-Life test: Age and sex differences. Journal of Clinical Psychology, 39 (3), 384-386.

Molasso, W. R. (2006). Exploring Frankl's Purpose in Life with college students. Journal of College and Character, 7 (1), 1-10.

Noblejas de la Flor, M. A. (1994). Logoterapia. Fundamentos, principios y aplicación. Una experiencia de evaluación del "logro interior de sentido". Tesis Doctoral, Universidad Complutense de Madrid, España.
Nygren, B., Aléx, L., Jonsén, E. Gustafson, Y., Norberg, A. \& Lundman, B. (2005). Resilience, sense of coherente, purpose in life and self-transcendence in relation to perceived physical and mental health among the oldest old. Aging \& Mental Health, 9 (4), 354-362.

Reker, G. T. (2000). Theoretical perspective, dimensions, and measurement of existential meaning. En G. T. Reker \& K. Chamberlain (Eds.), Exploring existential meaning: Optimizing human development across the life span (pp. 39-55). Thousand Oaks, CA: Sage.

Reker, G. T. \& Chamberlain, K. (2000). Existential meaning: Reflections and directions. En G. T. Reker \& K. Chamberlain (Eds.), Exploring existential meaning: Optimizing human development across the life span (pp. 199-209). Thousand Oaks, CA: Sage.

Sallee, D. T. \& Casciani, J. M. (1976). Relationship between sex drive and sexual frustration and purpose in life. Journal of Clinical Psychology, 32 (2), 273-275.

Schulenberg, S. E. (2003). Empirical research and logotherapy. Psychological Reports, 93, 307-319.

Sivberg, B. (2002). Coping strategies and parental attitudes: A comparison of parents with children with autistic spectrum disorders and parents with non-autistic children. International Journal of Circumpolar Health, 61 (2), 36-50.

Unger, R. K. (1979). Toward a redefinition of sex and gender. American Psychologist, 34 (11), 1085-1094.

Witkin, H. A. \& Goodenough, D. R. (1985). Estilos cognitivos: naturaleza y orígenes. Madrid: Pirámide. 


\section{Apéndice}

Purpose-In-Life Test [PIL] (versión de Noblejas de la Flor, 1994)

1. Generalmente me encuentro:

\begin{tabular}{|llllll|}
\hline 1 & 2 & 4 & 6 & 7 \\
Completamente aburrido & 3 & Neutral & 5 & 6 & Exhuberante, entusiasmado \\
\hline
\end{tabular}

2. La vida me parece:

\begin{tabular}{|llllll|}
\hline 1 & 2 & 3 & 4 & 6 & 7 \\
Completamente rutinaria & & Neutral & 5 & 6 & Siempre emocionante \\
\hline
\end{tabular}

3. En la vida tengo:

\begin{tabular}{|c|c|c|c|c|c|c|}
\hline $\begin{array}{l}1 \\
\text { Ninguna meta o anhelo }\end{array}$ & 2 & 3 & $\begin{array}{l}4 \\
\text { Neutral }\end{array}$ & 5 & 6 & $\begin{array}{l}7 \\
\text { Muchas metas y anhelos } \\
\text { definidos }\end{array}$ \\
\hline
\end{tabular}

4. Mi existencia personal es:

\begin{tabular}{|c|c|c|c|c|c|c|}
\hline $\begin{array}{l}1 \\
\text { Sin sentido ni propósito }\end{array}$ & 2 & 3 & $\begin{array}{l}4 \\
\text { Neutral }\end{array}$ & 5 & 6 & $\begin{array}{l}7 \\
\text { Llena de sentidos y propó- } \\
\text { sitos }\end{array}$ \\
\hline
\end{tabular}

5. Cada día es:

\begin{tabular}{|llllll|}
\hline 1 & 2 & 3 & 6 & 7 \\
Exactamente igual & 3 & Neutral & 5 & 6 & Siempre nuevo y diferente \\
\hline
\end{tabular}

6. Si pudiera elegir:

\begin{tabular}{|c|c|c|c|c|c|c|}
\hline $\begin{array}{l}1 \\
\text { Nunca habría nacido }\end{array}$ & 2 & 3 & $\begin{array}{l}4 \\
\text { Neutral }\end{array}$ & 5 & 6 & $\begin{array}{l}7 \\
\text { Tendría otras nueve vidas igua- } \\
\text { les a ésta }\end{array}$ \\
\hline
\end{tabular}

7. Después de retirarme:

\begin{tabular}{|c|c|c|c|c|c|c|}
\hline $\begin{array}{l}1 \\
\text { Holgazanearía el resto de } \\
\text { mi vida }\end{array}$ & 2 & 3 & $\begin{array}{l}4 \\
\text { Neutral }\end{array}$ & 5 & 6 & $\begin{array}{l}7 \\
\text { Haría las cosas emocionantes } \\
\text { que siempre deseé realizar }\end{array}$ \\
\hline
\end{tabular}

8. En el logro de mis metas vitales:

\begin{tabular}{|llllll|}
\hline 1 & & 4 & & 7 \\
$\begin{array}{l}\text { No he conseguido ningún } \\
\text { progreso }\end{array}$ & 3 & Neutral & 5 & 6 & $\begin{array}{l}\text { He llegado a mi realización } \\
\text { completa }\end{array}$ \\
\hline
\end{tabular}

9. Mi vida es:

\begin{tabular}{|llllll|}
\hline 1 & & 4 & & 7 \\
$\begin{array}{l}\text { Vacía y llena de desespe- } \\
\text { ración }\end{array}$ & 3 & Neutral & 5 & 6 & $\begin{array}{l}\text { Un conjunto de cosas buenas } \\
\text { y emocionantes }\end{array}$ \\
\hline
\end{tabular}


10. Si muriera hoy, me parecería que mi vida ha sido:

\begin{tabular}{|lllllll|}
\hline 1 & 2 & 3 & 4 & 6 & 7 \\
Una completa basura & 2 & & Neutral & 5 & 6 & Muy valiosa \\
\hline
\end{tabular}

11. Al pensar en mi propia vida:

\begin{tabular}{|llllll|}
\hline $\begin{array}{l}1 \\
\begin{array}{l}\text { Me pregunto a menudo la } \\
\text { razón por la que existo }\end{array}\end{array}$ & 3 & $\begin{array}{lllll}7 \\
\text { Neutral }\end{array}$ & 5 & 6 & $\begin{array}{l}\text { Siempre encuentro razones } \\
\text { para vivir }\end{array}$ \\
\hline
\end{tabular}

12. Tal y como yo lo veo en relación con mi vida, el mundo:

\begin{tabular}{|c|c|c|c|c|c|c|}
\hline $\begin{array}{l}1 \\
\text { Me confunde por com- } \\
\text { pleto }\end{array}$ & 2 & 3 & $\begin{array}{l}4 \\
\text { Neutral }\end{array}$ & 5 & 6 & $\begin{array}{l}7 \\
\text { Se adapta significativa mente } \\
\text { a mi vida }\end{array}$ \\
\hline
\end{tabular}

13. Me considero:

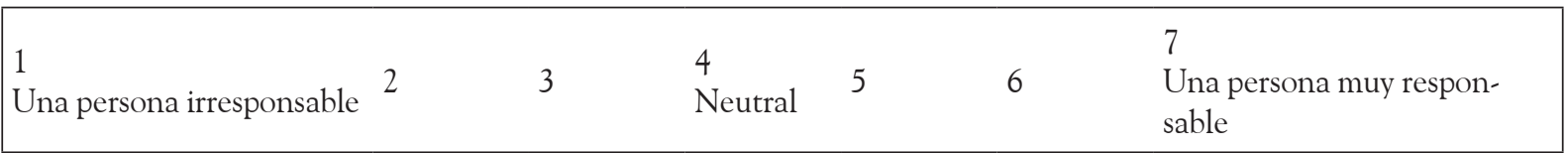

14. Con respecto a la libertad de que dispone para hacer sus propias elecciones, creo que el hombre es:

\begin{tabular}{|lllllll|}
\hline $\begin{array}{l}\text { Completamente esclavo } \\
\text { de las limitaciones de la } \\
\text { herencia y del ambiente }\end{array}$ & 2 & 3 & 4 & 5 & 6 & $\begin{array}{l}\text { Absolutamente libre de hacer } \\
\text { todas sus elecciones vitales }\end{array}$ \\
\hline
\end{tabular}

15. Con respecto a la muerte, estoy:

\begin{tabular}{|lllllll|}
\hline $\begin{array}{l}1 \\
\begin{array}{l}\text { Falto de preparación y } \\
\text { atemorizado }\end{array}\end{array}$ & 2 & 3 & $\begin{array}{l}4 \\
\text { Neutral }\end{array}$ & 5 & 6 & $\begin{array}{l}7 \\
\text { Preparado y sin temor }\end{array}$ \\
\hline
\end{tabular}

16. Con respecto al suicidio:

\begin{tabular}{|c|c|c|c|c|c|c|}
\hline $\begin{array}{l}1 \\
\text { Lo he considerado seria- } \\
\text { mente como una salida a } \\
\text { mi situación }\end{array}$ & 2 & 3 & $\begin{array}{l}4 \\
\text { Neutral }\end{array}$ & 5 & 6 & $\begin{array}{l}7 \\
\text { Nunca le he dedicado un } \\
\text { segundo pensamiento }\end{array}$ \\
\hline
\end{tabular}

17. Considero que mi capacidad para encontrar un significado, un propósito o una misión en la vida es:

\begin{tabular}{|lllllll|}
\hline 1 & 2 & 3 & 4 & 6 & 7 \\
Prácticamente nula & 2 & Neutral & 5 & 6 & Muy grande \\
\hline
\end{tabular}

18. Mi vida está:

\begin{tabular}{|lllllll|}
\hline $\begin{array}{l}\text { Fuera de mis manos y } \\
\text { controlada por factores } \\
\text { externos }\end{array}$ & 2 & 3 & 4 & 5 & 6 & $\begin{array}{l}7 \\
\text { En mis manos y bajo mi } \\
\text { control }\end{array}$ \\
\hline
\end{tabular}


Joaquín García-Alandete, Eva Rosa Martínez, Beatriz Soucase Lozano, José Francisco Gallego-Pérez

19. Enfrentarme a mis tareas cotidianas supone:

\begin{tabular}{|c|c|c|c|c|c|}
\hline $\begin{array}{l}1 \\
\text { Una experiencia dolorosa } \\
\text { y aburrida }\end{array}$ & 2 & $\begin{array}{l}4 \\
\text { Neutral }\end{array}$ & 5 & 6 & $\begin{array}{l}7 \\
\text { Una fuente de placer y satis- } \\
\text { facción }\end{array}$ \\
\hline
\end{tabular}

\section{He descubierto:}

\begin{tabular}{|c|c|c|c|c|c|}
\hline $\begin{array}{l}1 \\
\text { Ninguna misión o propósi- } 2 \\
\text { to en mi vida }\end{array}$ & 3 & $\begin{array}{l}4 \\
\text { Neutral }\end{array}$ & 5 & 6 & $\begin{array}{l}7 \\
\text { Metas claras y un propósito } \\
\text { satisfactorio para mi vida }\end{array}$ \\
\hline
\end{tabular}

